\title{
Implementing 2DT on a Multiprocessor
}

\author{
Yosi Ben-Asher \\ Haifa University \\ yosi@cs.huji.ac.il
}

\author{
Gudula Rünger Reinhard Wilhelm \\ Universität des Saarlandes \\ \{ruenger,wilhelm\}@cs.uni-sb.de
}

\author{
Assaf Schuster \\ Technion IIT \\ assafs@cs.technion.ac.il
}

\begin{abstract}
The implementation of a parallel functional language is discussed. 2DTprograms are composed of local SPMD-computations and global transformations of 2-dimensional data structures leading to a coarse grain computecommunicate scheme. The implementation is made up of virtual processes doing the local computations and abstract processors emulating several virtual processes and taking care of communication. Code and data structures which are common to virtual processes are shared and allocated to abstract processes in order to make context switches cheap. Code that would be executed redundantly by logical processes is converted to abstract code and executed only once. Aims of this effort are maximizing the amount of parallelism, keeping communication cheap and managing parallelism efficiently.
\end{abstract}

\section{Introduction}

2DT (2-dimensional transformations) is a general paradigm for the design of parallel SPMD (single-program-multiple-data) languages for multiprocessor systems proposed in [BRSW93a]. 2DT programs are composed of two levels of computation, local computations on local data and global transformations on ordered combinations of local data (2D-arrays). The local computations are performed by an existing sequential base language and modify all local data in a SPMD-ic fashion. For the parallel functional language 2DT-FP, Backus FP [Bac78] is chosen to be the base language [BRSW93a]. The global transformations serve as a communication mechanism. They may reshape a $2 \mathrm{D}$-array thereby rearranging its components, exchanging the components of a $2 \mathrm{D}$-array, splitting one $2 \mathrm{D}$-array into several new $2 \mathrm{D}$-arrays, or reglueing some 2D-arrays together. Thus, 2DT programs reflect a coarse grain compute-communicate scheme which is appropriate for the formulation of loosely synchronous algorithms which, e.g., arise in numerical problems. In addition to the parallelism on local data, operations on different 2D-arrays can be performed in parallel. Thus, a high degree of parallelism is provided.

An implementation of 2DT on a distributed multiprocessor network should be efficient and should preserve the degree of parallelism. We will achieve this by an implementation with the following design goals:

- Maximal amount of parallelism

- Cheap communication

- Efficient management of parallelism

- Portability

- Efficiency on the target machine

For the implementation, we associate each local data with a virtual process. Such a virtual process performs the local (FP) computations and, at least conceptually, is 
responsible for the communication realizing its part of global transformations. Virtual processes are executed by abstract processors each running on one physical processor. Abstract processors have to do virtual process management, i.e. creation, deletion, allocation, and scheduling of virtual processes. We will see that for efficiency reasons they also take over communication from their respective virtual processes. The group parallelism is handled by process group management, i.e. allocation, scheduling of groups of processes.

In order to exploit high amount of parallelism and since array sizes are dynamic, we have to employ dynamic load-balancing. But due to the regular SPMD-mode, load-balancing is relatively simple. Cheap communication is attained by the combination of small messages to large ones. This is feasible because in communication stemming from global transformations lots of local data are involved. Efficient management of parallelism is achieved by context sharing of virtual processes and clever process group management. Context sharing means that all the common components of the virtual processes of a 2D-array are shared leaving very small contexts to be switched. The implementation of global transformations consists in a topology independent message-preparation phase to be performed on each single abstract processor and a communication phase between the abstract processors. This scheme supports portability for the preparation phase. Efficiency is achieved by using specific communication algorithms provided on the physical target machine.

FP-type functional languages are attractive for parallel implementation and for parallel programming. The apply-to-all-operator can easily be given a parallel implementation. This is essentially, what has been done in the implementation of FP* [BW91]. However, FP*-programs are translated into some data parallel version of Fortran. No direct, efficient implementation on a multicomputer is realized.

[DP93] describe an interesting approach to the parallel implementation of FP. They correctly argue, that the code generated for a function should depend on the way, the data are distributed, on which the function operates. There are only a fixed set of functionals in FP. Thus, [DP93] propose to define a code template for essentially any way a functional can be used. This template contains an analytical formula for the costs connected with executing the corresponding code. A complex compiler organization is described to find out the best combination of templates for a given FP program. The approach looks nice, but hasn't been tested in practice. In addition, the templates have only static parameters including the problem size. This we consider unrealistic.

The equivalent of apply-to-all in lazy functional languages is map. It has also been regarded as a natural parallel operator [Hil93][Jou91]. Both papers consider the compilation of functional languages with aggregate data types and corresponding map functions to SIMD architectures. The stated assumption, that any monolithic operation, whose individual operation takes $O(1)$, when applied to on an aggregate of size $n$ executed on a SIMD computer of size $p$ takes also constant time, is not shared by these authors. Working on the increasingly popular MIMD machines under more realistic assumptions about execution and communication times is of great importance.

The paper is organized as follows. Section 2 introduces the general 2DT-paradigm and the language 2DT-FP. The association of local data with virtual processes is described in section 3 . Section 4 contains allocation strategies and the abstract machine model. The code-generating function is given in section 5. Finally, section 6 presents some 2D-transformation and their intended communication behavior. 


\section{Overview of the Language 2DT-FP}

The 2DT-Paradigm : The essential concept of 2DT-languages is a two-level hierarchy of local and global computations which are performed on parts or on the entire set of the problem-data. The execution of a 2DT-program $\mathcal{P}$ consists of a sequence of consecutive operations $\mathcal{P}=T_{k} ; \ldots ; T_{1}$ in which local and global functions alternate. The semicolon ";" denotes function composition. A 2DT-program $\mathcal{P}$ is applied to problem data which are divided into disjoint parts $L_{i}, i=0, \ldots, m-1$, called local data $(L o D a)$. The whole set of problem data is represented as a tuple of local data, i.e. $L=\left(L_{0}, \ldots, L_{m-1}\right)$. Depending on the currently executed local or global function of the program $\mathcal{P}$ the view on the data is different.

Local computations are performed on the lower level of the data representation by applying the same local function $F$ to each component $L_{i}$ of the data-tuple in parallel, see Fig.1a (apply-to-all parallelism). The data-structure of the local data ( $L o D a$ ) is inherited from the original data according to the considered algorithm, e.g. input data forming a matrix may be subdivided into blocks with two-dimensional structure or columns with one-dimensional structure.

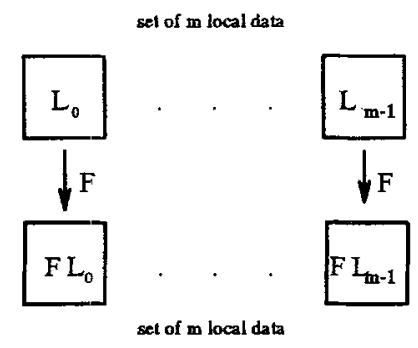

a) Local computation $F$

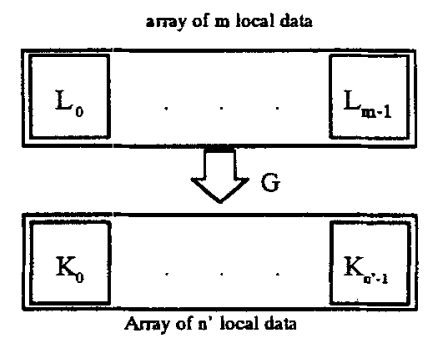

b) Global transformation $G$

Figure1: 2DT computations

Global transformations $G$ are applied to the upper level, the entire tuple of data $L$, see Fig.1b. In contrast to local computations, where we don't assume a special form of the $\operatorname{LoDa} L_{i}$, 2-dimensional transformations are only defined on 2dimensional (rectangular) data-structures. Thus, all local data $L_{i}$ are described as linear data $L_{i}=\left(L_{i}^{1}, \ldots, L_{i}^{n}\right)$ of the same length $n$. The structure of the data $L_{i}^{j}$ is left unspecified in the general case and has to be designed according to the special algorithm. The two-dimensional rectangular "data in a global state" is called 2D-array of shape $(n, m)$ (see Fig.2).

Global transformations don't change the inner structure of the components $L_{i}^{j}$, but reorganize them into a new shape. According to their reshaping behavior three classes of transformations are considered:

(1) Shape-preserving transformations leave the shape of a $2 \mathrm{D}$-array unchanged. The data components $L_{i}^{j}$ of the current $2 \mathrm{D}$-array are moved to other places $\left(i^{\prime}, j^{\prime}\right)$ in the resulting $2 \mathrm{D}$-array.

(2) Reshaping transformations change the shape of the 2D-array in a prescribed way. The set of data $\left\{L_{i}^{j} \mid i=0, \ldots, m-1, j=1, \ldots, n\right\}$ of the current $2 \mathrm{D}$-array remains the same but the items $L_{i}^{j}$ are restructured into a new shape. 


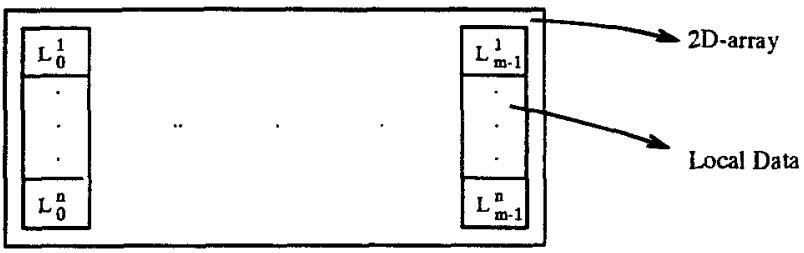

Figure2: Data in a global state of shape $(n, m)$

(3) Array-creating transformations change the number of the considered 2Darrays. For the creation, the LoDa $L_{i}$ of the current 2D-array are divided into disjoint subsets which form the new 2D-arrays. For the creation of one $2 \mathrm{D}$-array from a set of $2 \mathrm{D}$-arrays, the LoDa of the $2 \mathrm{D}$-arrays are joined. In both cases, the internal compositions of the LoDa's are unchanged. The use of array-creating transformations in a program is block-structured, i.e. a creation of several 2D-arrays is followed be a corresponding join operation. Different pairs of array-creating transformations are fully-nested.

Array-creating transformations provide the possibility to consider different 2D-arrays forming a partition of the local data. Local and global functions are applied to each single 2D-array as described for the entire set of local data. Those applications are performed independently and offer a second kind of parallelism, called array parallelism (see e.g. Fig.1, when la is performed in parallel with 1b).

The 2DT-paradigm includes arbitrary global transformation possessing one of the behaviors described above. Examples for each class of transformations are given in section 6. For each concrete (implemented) 2DT-language a fixed set of global transformations is provided. In this sense, $2 \mathrm{DT}$ describes a class of $2 \mathrm{DT}$-languages.

The language 2DT-FP: The functional language FP [Bac78] is chosen to express local computations. Thus, the $L o D a L_{i}$ and also the data components $L_{i}^{j}$ of data in a global state are FP-objects. The local functions are FP-functions which are executed on each component $L_{i}$ of a tuple of FP-objects $\left(L_{0}, \ldots, L_{m-1}\right)$ in parallel in SPMD-ic fashion producing a new tuple of FP-objects.

The execution of a program $\mathcal{P}$ essentially consisting of a sequence of local FP functions and global transformations is done by consecutively applying the functions to the problem data $L$ from the left to the right, i.e.

$$
\mathcal{P} L=\left(T_{k} ; \ldots ; T_{1}\right) L=T_{k}\left(\ldots\left(T_{1}(L)\right)\right)
$$

";" between local and global transformations also causes synchronization. For the exact syntax and semantics see [BRSW93a].

\section{$3 \quad$ Virtual Processes}

The local data $L_{i}, i=1, \ldots, m$, are associated with virtual processes with process numbers $i$. More precisely, the association of data with virtual processes means that computations on LoDa $L_{i}$ are performed by the corresponding process.

The global shape-preserving transformations, which move data across the LoDa of $2 \mathrm{D}$-arrays, serve as the communication mechanism between the virtual processes. 


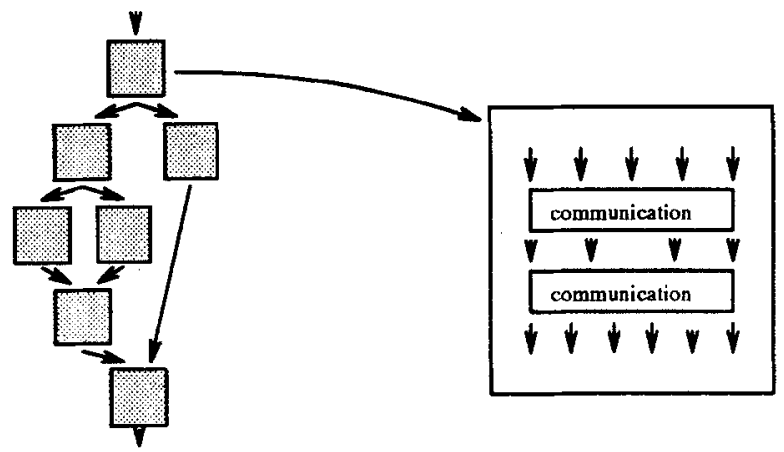
a) Example of global-task graph
b) Example of local-task graph

Figure3: Two-level task graph

Because of this special implicit description of communication, all virtual processes associated to one 2D-array take part in each communication phase. Synchronization has to ensure correct message passing for each transformation.

The creation of new 2D-array components via reshaping transformations extends the number of the current virtual processes for the next local computation. Thus, spawning and deletion of virtual processes is connected to these data-manipulations.

The array-creating transformations divide the processes into groups each performing different tasks, i.e. 2D-arrays are represented by groups of virtual processes. In each group, the virtual processes are numbered consecutively starting with 0 .

Because of the alternation of local computations and global transformations, 2DT is appropriate for the formulation of loosely-synchronous algorithms consisting of consecutive segments (phases) of independent but similar computations separated by communication phases (coarse grain compute-communicate algorithm). In each phase, a process uses data received from other processes in the last communication phase. Changing the number of virtual processes by reshaping transformations allows to fully exploit the intrinsic parallelism of an algorithm on the virtual level.

The task graph is the conceptual structure representing the execution of a dynamically changing set of virtual processes. Within each single group, the local-task graph is structured into phases of computations and phases of communication with a variable dynamic number $n_{i}$ of tasks due to reshaping. Due to array-creating transformations, those homogeneous local-task graphs are arranged in a group-task graph of more general unbalanced structure. Figure 3 shows an example of a group-task graph where the nodes are local-task graphs and the arrows symbolize array-creating transformations. We consider two alternatives to handle group-parallelism:

- Depth first, i.e. the group-task graph is traversed with a depth first strategy.

- Breadth first, i.e. the group-task graph is traversed with a breadth first strategy. 


\section{Abstract Machine}

The next step from the idealized view of a variable number of virtual processes to an implementation on a processor-network with a fixed number of physical processors, i.e. from an infinite virtual parallelism to a finite one, is the mapping of a variable number of virtual processes to a fixed number of processors. To this end, we consider an abstract machine model consisting of a fixed number $p$ of abstract processors.

An allocation from virtual processes to abstract processors is associated with the basic problems of distributed computing:

load balancing, virtual process migration, group management.

It is in the nature of this basic problems that the best strategy for one of the problems may contradict the best solution to the others, e.g. good load balancing may cause lots of virtual process migration between abstract processors, or cheap group management may entail bad load balancing and much virtual process migration. Thus, an acceptable allocation strategy has to be a compromise between the competing distribution problems. On the other side, the allocation decision influence and restrict the run-time functions:

scheduling, change of allocation, communication between abstract processors. Thus, the allocation is the link between the conceptual strategy for solving the basic problems and the achievable run-time behavior. We provide an allocation mechanism representing the chosen allocation strategy in an appropriate data-structure VAPT which is used for the run-time functions. The abstract machine supports this separation between strategy and execution, and the abstract machine code contains the data structure VAPT. Several allocation strategies are discussed.

\subsection{Processor Allocation}

The abstract processors $P_{0}, \ldots, P_{p-1}$ are numbered consecutively but there is no constraint to a special topology of the network, in particular not to a processor-array. Each virtual process when created is allocated to an abstract processor. Because of the definition of virtual processes this comprises the distribution of both, the data and the respective computation work.

The allocation of all current virtual processes to abstract processors is described by an allocation function. Because of the varying number of virtual processes, the allocation mapping is not fixed but may change due to global transformations. Therefore, in the course of program execution, we will have a sequence of allocation functions $\quad \mathcal{A}_{t}:\left\{0, \ldots, n_{t}-1\right\} \longrightarrow\{0, \ldots, p-1\}, \quad t=1,2, \ldots$

where $n_{t}$ is the current number of virtual processes. The period in which one allocation function $\mathcal{A}_{t}$ holds is called allocation phase $t$ and the change to a new allocation phase $t+1$ ( a new allocation function $\mathcal{A}_{t+1}$ ) is referred to as reallocation. The virtual process $j$ existing in allocation phase $t$ is executed on the abstract processor $\mathcal{A}_{t}(j), j=0, \ldots, n_{t}-1$. During a local computation the allocation function is fixed. The allocation may change after a global transformation. But, each allocation phase may cover several computation and communication phases.

The current allocation information is stored in a table, the VAPT (virtual-toabstract-processor table) providing the correct allocation function $\mathcal{A}_{t}$ in the phases of local computation and in subsequent communications. As described before, several groups of virtual processes may exist at the same time. There is one VAPT ${ }_{g}$ to 
describe the allocation information for each group $g$. Thus, in case of a set of groups we have a set of VAPT's each of which stores the allocation mapping for one group of virtual processes.

From the point of view of each abstract processor in a given allocation phase $t$, the set of virtual processes is divided into internal and external processes. The set Internal $_{t}=$ Internal $_{t}\left(P_{l}\right)=\left\{k \mid \mathcal{A}_{t}(k)=l\right\}, \quad(l=0, \ldots, p-1)$, contains the process numbers of the internal virtual processes of $P_{l}$ within phase $t$; all other currently existing virtual processes are external processors for $P_{l}$. There is a partition of internal virtual processes according to group membership.

We will experiment with several simple allocation strategies which avoid frequent reassignments of virtual processes to abstract processors (virtual process migration), while keeping a balanced distribution of virtual processes on the abstract ones.

The 2DT-paradigm offers the following simple allocation situation:

- Independent tasks are directly expressed in the 2DT-program due to 2DT-and group-parallelism.

- A homogenuous task graph is given because all local computation within the same group are of similar size. The execution time may still be different, because the programs may use process numbers. However, likelihood for this is small. Thus, we can rather safely work under the assumption that a balanced process distribution implies a balanced load distribution. A balanced process distribution can be established during the initial distribution phase and maintained by redistribution.

If the number of abstract processors is greater than the number of virtual processes the assignment is straightforward. However, we suppose that the number of virtual processes exceeds the number of abstract processors, i.e. many virtual processes will be executed by one abstract processor.

\section{Allocation strategies:}

(1) Random allocation:

The local data are distributed over the abstract processors at random. [BSW89] shows that with high likelyhood a good distribution can be achieved. However, locality is not guaranteed.

(2) Multifit algorithm for nonpreemptive tasks [CGJ78]:

The set of nonpreemptive tasks, as it is e.g. given for numerical algorithms, is distributed over the processors by an efficient approximation algorithm ([CGJ78]). The global execution time is at most by a factor 1,220 worse than the global execution time of an optimal allocation.

(3) $\left\lceil\frac{n}{p}\right\rceil$-consecutive allocation [BRSW93b]:

The $n$ local data of each 2D-array are evenly distributed over the abstract processors such that each holds at most $\left\lceil\frac{\pi}{p}\right\rceil$-consecutive local data of the array.

(CA) The number of LoDa allocated to abstract processors differs at most by one (New load-balancing is needed after $\log p$ resize operation (sec.6), see[BRSW93b])

(4) Group-oriented allocation:

The allocation of virtual processes partitions the set of physical processors according to the group structure of the virtual processes, i.e. the following invariance is always guarantees the:

(BF) No virtual processes of different $2 D$-arrays execute on the same processor This simplifies the communication implementing global transformations, since messages are exchanged only inside groups of virtual processes. 
(5) History-sensitive allocation:

Depending on the current allocation situation stored in the VAPT, a new allocation is incrementally computed avoiding too many process migration.

\subsection{Structure of Abstract Processors}

Conceptually, all virtual processes have identical copies of the translated 2DT-FP program. They apply this code to their local data. The semantics of the different copies, however, differ, since the code contains process numbers.

In an implementation, all internal virtual processes of one abstract processor share the same copy of the code. The abstract processor schedules them with the right processor number and the right local data. In the same way, all other common information are factored out and are part of the abstract processor (context sharing). All scheduling strategies discussed will also allow the sharing of the runtime stacks for function calls. This leads to the following structure of an abstract processor:

- the program,

- VAPT's, one for each group containing at least one internal virtual process;

- allocation phase information: (number of internal virtual processes, total number of virtual processes, address of the first internal process, height of LoDa.)

- input- and output-buffers for communication with other abstract processors,

- a run-time system supporting scheduling, synchronization, updating VAPT's and communication (external and inAbstract processor

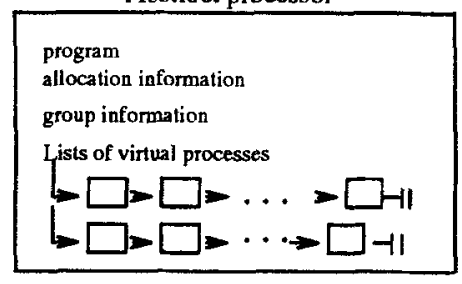
ternal restoring)

- lists of virtual process descriptions of internal processes (one for each group involved).

The concept of context sharing leaves small virtual process description. The representation of a virtual process is reduced to their individual components, especially to the local data and the virtual process number. Furthermore, information for local computations (e.g. size and address of local data) and information used for the scheduling by the abstract processor (e.g. program counter) are contained.

Group context sharing makes context sharing between processes of the same group cheap. This is essentially exploited by using the group-oriented allocation (4), where each abstract processor runs only virtual processes from one group. Thus, we have process migration in favour of cheap context switches.

\subsection{Abstract Machine Code}

The generated machine code has to perform both, the execution of virtual processes and the management of allocation. The following table contains 2DT-FP functions and the intended behavior of the translated program: 


\begin{tabular}{l|l} 
2DT-FP function in source program & Execution on the abstract processors \\
\hline FP-function & Execution for internal processes \\
Shape-preserving transformation & Redistribution of data \\
Reshaping transformation & Updating the VAPT \\
& Redistribution of data \\
Array-creating transformation & Creation or deletion of VAPT's \\
& Updating of VAPT's \\
& Redistribution of data
\end{tabular}

An identical updating of the VAPT's is performed by all abstract processors, according to the same chosen global allocation strategy. Creation or deletion of VAPT's reflect the creation or deletion of groups due to array-creating transformations.

\subsubsection{Communication}

Shape-preserving global transformations are translated into appropriate communication code. Therefore, the virtual communication messages (which can be created directly from the global transformations) have to be converted to abstract messages. Abstract processors realizing virtual communication perform communication between internal virtual processes by reshuffling the data in place. Abstract communication redistribute data of external processes (redistribution).

Reshaping and array-creating transformations give rise to the reallocation of abstract processors (and to the updating of the VAPT), i.e. the whole data-structure describing a virtual process is forwarded to another abstract processor.

According to redistribution and reallocation, we have to distinguish differnet kinds of atomic messages for abstract communication, e.g.:

- Redistribution message (Dist) for 2D-array component $L_{i}^{j}$ :

$$
\text { (Dist, processno, com, contents), }
$$

where contents is the value to be stored in the virtual process with number processno in local data component com.

- Reallocation message (Allo) for virtual process description with local data $L_{i}$ :

(Allo, processno, contents)

where contents is the virtual process description to be stored as virtual process processno.

For the abstract communication, we provide an appropriate set of input-buffers and output-buffers, i.e. each abstract processor $P_{l}$ possesses $p-1$ input-buffer $\mathcal{I}_{k}$ and $p-1$ output-buffers $\mathcal{O}_{k}, k \in\{0, \ldots, p-1\}-\{k\}$.

In order to realize the packing of large messages and to use efficient topologydependent communication primitives, each abstract processor splits the communication phases into three separate steps (communication bundling):

(1) preparation of atomic messages,

(2) abstract communication and

(3) extracting and distributing of atomic messages.

In detail in each step the following is performed:

(1) Redistribution messages are prepared according to the generating transformation. The abstract destination is determined with the help of the VAPT. The destination for a reallocation messages is also given by the VAPT updated according to the considered transformation. 
(2) After finishing the preparation step, the atomic messages in each output buffer $\mathcal{O}_{k}, k \in\{0, \ldots, p-1\}-\{k\}$ are combined to one large message. When all the local virtual processes have finished this, communication takes place. Because communication is caused by global transformations, every processor knows how many messages (at most $p-1$ ) it expects from this transformation.

If every abstract processor holds internal processors of each current groups, we have to perform a synchronous total exchange operation, see [BT89]. In case of group-oriented allocation, a synchronous total exchange operation is performed on subnets. The communication can also be expressed in terms of communication primitives, e.g. non-blocking send actions, blocking receive actions, $\mathrm{cf}$. [And91] which are available on most topologies as offered e.g. by [BBD ${ }^{+} 87$ ].

(3) The atomic messages of the received large messages are stored. Messages of type $D i s t$ are stored as the component corn of the local data of virtual process processno. Messages of type Allo are inserted in a list of virtual processes of the same group as process number processno.

\subsubsection{Scheduling - Group Management}

According to the two-level task graph (see Fig.3), the abstract machine has to perform two different kinds of scheduling.

Scheduling in the small: Local computations for internal processes within the the same group are scheduled by the abstract processor. The same is done for the local parts of shape-preserving global transformations. Scheduling will switch between virtual processes after local computations and the set up phase of messages.

Scheduling in the large: On the level of groups of virtual processes, scheduling is performed according to the chosen depth-first or breadth-first strategy.

The depth-first strategy admits only one active 2D-array at a time performed by scheduling in the small. Depth first group scheduling may severely restrict the amount of parallelism. Computation and communication phases are strictly sequential, thus potentially leaving processors idle durnig communication phases and the network idle during computation phases.

Breadth-first group scheduling strategy has to solve the problem that messages caused by several transformations may coexist in the network. Using the processor allocation strategy (4), the abstract processors are grouped according to the grouping of the virtual processes, i.e. no processor runs virtual processes from more than one group, because of invariance (BF). Hence, message exchange for each application of a transformation is restricted to one subnet. This strategy may better exploit the network, but it still leaves the processors idle during group communication phases.

A more general breadth first group scheduling offers the greatest degree of parallelism. Local computations of one group may be performed overlapping with communication phases of another group for each processor. However, this will lead to the situation, that one processor may receive messages from different transformation applications at the same time.

\subsubsection{Synchronization}

Synchronization is needed in parallel systems, if the relative speed of processes may influence the result of computations. This is mainly due to competing accesses to shared 
resources. 2DT-FP does not have shared or global variables. Furthermore, synchronization ensures that local computations take place on the correct data transmitted in the last communication phase.

Because within a local-task graph communication phases are restricted by the special global transformations, local synchronization (see [BT89]) is used, i.e. a processor knows from which other processor it has to expect messages and thus it starts a new computation once it has received all these messages. Hence, explicit synchronization is eliminated.

There is only one problem potentially needing synchronization. This is the coexistence of messages from different global transformations without clear indication of what was the transformation application which generated it.

In case of depth-first group scheduling strategy which admits only one active 2Darray at any time local synchronization can be exploited and no extra communication is required.

The breadth-first group scheduling together with group-oriented allocation strategy with invariant $(\mathrm{BF})$ restricts communication to a subnet. Hence, we also have local synchronization.

For breadth-first group management with general allocation, we have to solve the problem of identifying the right (virtual) receiver. A unique identification scheme for transformation applications is introduced which is described in detail in [BRSW93b]. This also leads to an implicit synchronization similar the one in the depth-first case. Every group member on one processor knows how many messages to expect. Again, no extra communication is required.

\section{Code Generation}

The code for the abstract machine is generated by the code-generating function $\mathcal{G}$. This function $\mathcal{G}$ translates a 2DT-FP programs into abstract machine code. The generated abstract code has to perform modifications on data-structures describing virtual processes. As mentioned before, all abstract processors execute identical copies of an abstract program. The semantics differ because of different internal processes Internal and different VAPT information. \# denotes the number of the virtual process currently executed.

We restrict the presentation to the translation of the global transformations in the message creating phase. Some functions used in the target code are:

\begin{tabular}{l|l} 
Function & Behavior \\
\hline schedule & scheduling of internal processes \\
update & $\begin{array}{l}\text { updating of the VAPT } \\
\text { store }\end{array}$ \\
storing data into the appropriate buffer or data-structure
\end{tabular}

(1) Let $T$ be a global shape-preserving transformation

schedule for \# $\in$ Internal $_{t}$ :

$\{$ For $j=1, \ldots, n$ do

$m s g=$ create-msg $(T, j)$;

if $(\operatorname{VAPT}(\operatorname{dest}(m s g))=$ own abstract processor number $)$

then store $(m s g, \operatorname{dest}(m s g))$

else store $\left(m s g, O_{V A P T(\operatorname{dest}(m s g))}\right)$

endif $\}$ 
where create-msg $(T, j)$ is a macro creating the destination of a message component $j$ for Transformation $T$, e.g. for the transpose transformation $c 2 r$ (cf. sec.6) we have:

create-msg $(\mathrm{c} 2 \mathrm{x}, j)=\left(\right.$ Dist $\left.,(\# * n+j-1) \bmod m,(\# * n+j-1) \operatorname{div}+1, L_{\#}^{j}\right)$

(2) Let $T$ be a global reshaping transformation (reallocation from $t$ to $t+1$ ):

update $(T)$ VAPT ;

rename virtual processes $(T)$

create (or delete) processes $(T)$

schedule for \# $\in$ Internal $:$

$\{m s g=$ create-msg $(T, j)$;

if $(V A P T(\operatorname{dest}(m s g))=$ own abstract processor number $)$

then store $(m s g$, dest $(m s g))$

else store $\left(m s g, O_{V A P T(\text { dest }(m s g))}\right)$

endif $\}$

(3) Let $T$ be a global array-creating transformation (for group oriented allocation):

create (or delete) VAPT's $(T)$

rename virtual processes $(T)$

schedule for \# $\in$ Internal $t$

$\left\{\right.$ if $\left(\mathcal{A}_{t} \neq \operatorname{VAPT}(\#)\right)$

then $m s g=$ create-msg $(T, \#)$;

store $\left(m s g, O_{V A P T(\text { dest(msg)) }}\right)$

endif $\}$

The application of a program to the problem data is performed by the apply command. Before running the program, the expected situation on the abstract processors has to be initialized:

(a) Each abstract processor builds up its internal structure with an empty list of virtual processes.

(b) The program is loaded into the program store.

(c) According to the problem data division, the first allocation function $\mathcal{A}_{1}$ is determined and stored.

(d) The virtual processes are initialized according to the VAPT .

(e) The local data are stored in their corresponding virtual structure.

\section{Examples of Global Transformations}

In this chapter, we present transformations proposed in [BRSW93a] and illustrate their communication behavior on the virtual level.

\subsection{A Shape-Preserving Transformations: Transpose}

The transpose operation columns-to-rows (c2r) applied to a rectangular 2D-array changes the data storage in column-major order to row-major order. The transformation rows-to-columns ( $\mathrm{r} 2 \mathrm{c}$ ) performs the opposite.

\begin{tabular}{|c|c|c|c|c|c|c|c|c|c|c|}
\hline $\begin{array}{l}\text { virsual } \\
\text { procaso }\end{array}$ & 0 & 1 & $\cdots$ & $m-1$ & & $\begin{array}{l}\text { vissual } \\
\text { proces! }\end{array}$ & 0 & 1 & $\ldots$ & $m-1$ \\
\hline 1 & $a_{1}$ & $a_{n+1}$ & & $a_{(m-1) n+1}$ & $52 c$ & 1 & $a_{1}$ & $a_{2}$ & $\cdots$ & $a_{m}$ \\
\hline$\vdots$ & $\vdots$ & $\vdots$ & & $\vdots$ & & $\vdots$ & $\vdots$ & $\vdots$ & & $\vdots$ \\
\hline$n$ & $a_{n}$ & $a_{2 n}$ & $\cdots$ & $a_{m n}$ & & $n$ & $a_{(n-1) m+1}$ & $a_{(n-1) m+2}$ & $\cdots$ & $a_{n m}$ \\
\hline
\end{tabular}


The transformation $\mathrm{c} 2 \mathrm{r}$ re-distributes the contents $a_{i * n+j}$ of LoDa $L_{i}^{j}$ into the LoDa of the virtual processor with the number

as tuple component with number

$$
(i * n+j-1) \bmod m \quad \text { for } i=0, \ldots, m-1, j=1, \ldots, n
$$

$(i * n+j-1)$ div $m+1 \quad$ for $i=0, \ldots, m-1, j=1, \ldots, n$.

Thus, each virtual process \# $\in\{0, \ldots, m-1\}$ has to send tuple component $j$ to the virtual process \#' with number

$$
\#^{\prime}=(\# * n+j-1) \bmod m \quad \text { for } j=1, \ldots, n
$$

which depends on its own processor number \#. Therefore each abstract processor creates the messages

$\left(\right.$ Dist $\left.,(\# * n+j-1) \bmod m,(\# * n+j-1) \operatorname{div}+1, L_{\#}^{j}\right)$ of type Dist which take part in a communication phase described in section 4.3.

The transformation r2c re-distribute the contents $a_{(j-1) * m+i+1}$ of LoDa $L_{i}^{j}$ into the LoDa of the virtual processor with the number

$$
(i+(j-1) * m) \text { div } n \quad \text { for } i=0, \ldots, m-1, j=1, \ldots, n
$$

as tuple component with number

$$
(i+(j-1) * m) \bmod m+1 \quad \text { for } i=0, \ldots, m-1, j=1, \ldots, n \text {. }
$$

The atomic messages are formed analogously to the transformation $c 2$ r.

\subsection{A Reshaping Transformations: Resize}

Resize(2) or Resize(-2)-transformations "double" or "half" the number of virtual processes and re-distribute the data in the following way. For simplicity, let $n$ and $m$ be even.

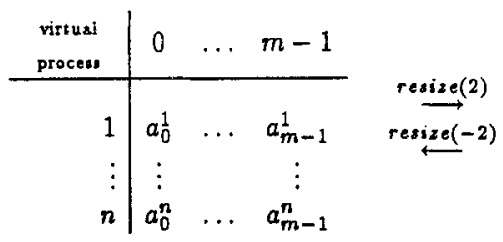

\begin{tabular}{r|ccccc} 
virtual & 0 & 1 & $\ldots$ & $2 m-2$ & $2 m-1$ \\
\hline procests & 0 & & & \\
1 & $a_{0}^{1}$ & $a_{0}^{[n / 2]+1}$ & $\ldots$ & $a_{m-1}^{1}$ & $a_{m-1}^{[n / 2]+1}$ \\
$\vdots$ & $\vdots$ & $\vdots$ & & $\vdots$ & $\vdots$ \\
$n$ & $a_{0}^{[n / 2\rceil}$ & $a_{0}^{n}$ & $\ldots$ & $a_{m-1}^{[n / 2]}$ & $a_{m-1}^{n}$
\end{tabular}

In order to perform a resize(2)-transformation, each abstract processor performs:

- The VAPT is extended and updated.

- The processor numbers of the existing virtual process \# are renamed in \#'= $2 * \#, \#=0, \ldots, m-1$.

- The virtual processes with number $\#^{\prime}=2 * l+1, l=0, \ldots, m-1$ are created in the appropriate abstract processor determined in the VAPT.

- The contents $a_{\#}^{[n / 2]+1}, \ldots, a_{\#}^{n}$ of the renamed virtual process $\#^{\prime}=2 * \#, \#=$ $0, \ldots, m-1$ are sent to the virtual processors with the numbers $\#^{\prime}+1$ in order to be the contents of $L-\#^{\prime}=L_{\#^{\prime}=1}^{1}, \ldots, L-\#^{\prime}+1^{[n / 2]}$.

- The 'length of the LoDa' is updated. 'The 'number of processes' is updated.

\subsection{An Array-Creating Transformations: Split\&Glue}

Split\&Glue transformation splits the 2D-array into two son arrays. The operation of Split\&Glue is determined by some arbitrary boolean condition that is applied to all columns: Those having the same result (True or False) are grouped together in the same son array. A separate and parallel computation (supplied as an argument 
to the constructors) is performed on every son array. When both computations have terminated, their results (two new 2D-arrays) are joined back forming a single 2Darray.

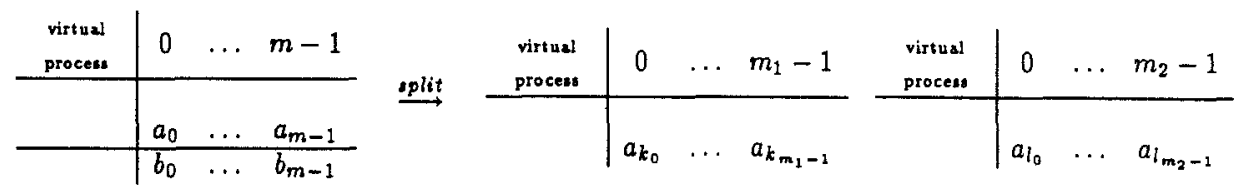

where $\quad b_{i} \in\{$ True, False $\}, i=0, \ldots, m-1, m_{1}+m_{2}=m$

and $b_{k_{\kappa}}=$ True, $\kappa=1, \ldots, m_{1}-1 b_{l_{\nu}}=$ False, $\nu=1, \ldots, m_{2}-1$.

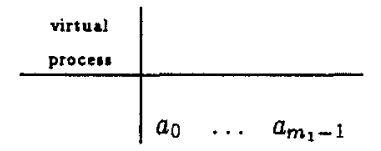

\begin{tabular}{cr|cccccc} 
& $\begin{array}{c}\text { virtual } \\
\text { process }\end{array}$ & 0 & $\ldots$ & $m_{1}-1$ & $m_{1}$ & $\ldots$ & $m_{1}+m_{2}-1$ \\
\hline & & & & & & \\
glue & 1 & $a_{0}^{1}$ & $\ldots$ & $a_{m_{1}-1}^{1}$ & $b_{0}^{1}$ & $\ldots$ & $b_{m_{3}-1}^{1}$ \\
& $\vdots$ & $\vdots$ & & $\vdots$ & $\vdots$ & & $\vdots$ \\
& $n^{\prime}$ & & & & $b_{0}^{n^{\prime}}$ & $\ldots$ & $b_{m_{2}-1}^{n^{\prime}}$ \\
& $\vdots$ & $\vdots$ & & $\vdots$ & $\vdots$ & & $\vdots$ \\
& $n$ & $a_{k_{0}}^{n}$ & $\ldots$ & $a_{m_{1}-1}^{n}$ & $@$ & $\ldots$ & $@$
\end{tabular}

where $n \geq n^{\prime}$ and @ is a null element (cf.[BRSW93a]). Depending on the allocation strategy, communication with messages of type Allo take place or not.

\section{Conclusion}

The 2DT paradigm provides a two-level parallelism of so-called apply-to-all parallelism and group parallelism. For the implementation we discussed several allocation strategies appropriate for the regular SPMD-mode of 2DT providing a course grain compute communicate scheme with regard to our design goals for distributed programming. Special emphasize was laid on group management handling group-parallelism. The design goals are achieved by the concepts of restricted dynamic allocation, packing of messages, context sharing and communication bundling.

The introduced implementation concept has an open structure concerning implementation decisions and also language design decisions. Thus, it allows the exchange of allocation strategies and communication concepts. As well, the implementer can add new 2D-transformation to an existing 2DT-implementation.

Currently, we are implementing 2DT-FP on the iPSC/860. We will experiment with the introduced allocation strategies using different kinds of test programs from e.g. numerical algorithms. This is connected with the design of distributed algorithms for (numerical) application and the study of usable 2D-transformation in the applications. Furthermore, the implementation concept will be applied to an imperative base language.

\section{References}

[And91] G.R.. Andrews. Paradigms for process interaction in distributed programs. ACM Computing Surveys, 23(1):49-90, 1991. 
[Bac78] J. Backus. Can programming be liberated from the von Neumann style? A functional style and its algebra of programs. Communication of the ACM, 21(8):613-641, 1978.

[BBD+87] J. Boyle, R. Butler, T. Disz, B. Glickfeld, E. Lusk, R. Overbeek, J. Patterson, and R. Stevens. Portable Programs for Parallel Processors. Holt, Rinehart and Winston, New York NY, USA, 1987.

[BRSW93a] Y. Ben-Asher, G. Rünger, A. Schuster, and R. Wilhelm. 2DT-FP: An FP based programming language for efficient parallel programming of multiprocessor networks. In PARLE'93, LNCS 694, pages 42-55. Springer Verlag, 1993.

[BRSW93b] Y. Ben-Asher, G. Rünger, A. Schuster, and R. Wilhelm. Load balancing and communication for a parallel functional language. Submitted for publication, 1993.

[BSW89] Y. Ben-Asher, H. Seidl, and R. Wilhelm. The TRANSPOSE machine: A global implementation of a parallel graph reducer. In Proc. of 'Tencon'89, Bombay, 1989.

[BT89] D.P. Bertsekas and J.N. Tsitsiklis. Parallel and Distributed Computing. Prentice Hall, 1989.

[BW91] D. Banerjee and C. Walinsky. An optimizing compiler for $\mathrm{FP}^{*}-$ a dataparallel dialect of FP. Parallel and Distr. Proc., pages 70-78, 1991.

[CGJ78] E.G. Coffman, M.R. Garey, and D.S. Johnson. An Application of BinPacking to Multiprocessor Scheduling. SIAM Journal of Computing, $7(1): 1-17,1978$.

[DP93] M. Danelutto and S. Pelagatti. Parallel implementation of FP using a template-based approach. In M. van Eekelen R. Plasmeijer, editor, Implementation of Functional Languages, pages 7-22, Nijwegen, Sept. 1993.

[Hil93] J. Hill. Vectorizing a non-strict functional language for a data-parallel "Spineless (not so) Tagless G-machine". In M. van Eekelen R. Plasmeijer, editor, Implementation of Functional Languages, pages 87-100, Nijwegen, Sept. 1993.

[Jou91] G.K. Jouret. Compiling functional languages for SIMD architectures. Parallel and Distributed Processing, pages 79-86, 1991. 